\title{
Filaggrin Null-Mutation in Asthma in an Indian Cohort: One Link in a Polygenic Trait
}

\author{
Ratna Dua Puri ${ }^{1}$ (D) \\ Received: 30 June 2020 / Accepted: 30 June 2020 / Published online: 10 July 2020 \\ (C) Dr. K C Chaudhuri Foundation 2020
}

On one end of a spectrum are monogenic disorders with allergic phenotypes that are caused by highly penetrant but rare gene mutations e.g., STAT3 related hyper-IgE syndrome. At the other end are complex, multifactorial disease phenotypes associated with low penetrance and common risk allele frequency that have an intermediate and very low disease effect.

What is the genetic background of the chronic inflammatory disease, asthma as well as other allergic disorders? It's heritable nature has been demonstrated in multiple studies and genetic factors are reported to have an impact on the susceptibility and severity of asthma in $60-70 \%$ patients. Hence environmental triggers on a backdrop of a genetic predisposition contribute to the development and progression of the disease.

Asthma and allergic disorders commonly occur in the same individual or within members of a family. Questions of a "disease continuum" or distinct disorders with individual etiologies have often been debatable. The basic "atopic march", recognized as a series of progressive clinical manifestations of atopy, is well described with primary sensitization in infancy and childhood [1].

Filaggrin; FLG [\#OMIM 135940], in the epidermis has an essential role in forming a protective skin barrier. Association of FLG with ichthyosis vulgaris was described in 2006 and subsequently its association and susceptibility for atopic dermatitis was suggested [2]. There is sensitization to food or aeroallergens in early life with progression to eczema and wheezing, often followed by chronic asthma, allergic rhinitis and other atopic disorders. As $70 \%$ patients with atopic dermatitis develop asthma, it was logical that FLG be probed for its association with asthma.

Ratna Dua Puri

ratnadpuri@yahoo.com

1 Institute of Medical Genetics and Genomics, Sir Ganga Ram Hospital, Rajinder Nagar, New Delhi 110060, India
In the current study published in this issue of IJP, the authors hypothesized that the common mutations in FLG could be related to allergic disorders in Indian children [3]. This prospective, case control study, tested the R501X mutation in 90 enrolled children with asthma alone or along with atopic dermatitis and/or rhinitis. Though they did identify a mutant homozygous genotype in $5.5 \%$ children with asthma and/or asthma with eczema, it was not statistically significant.

Ethnic differences for FLG mutations are well reported and multiple reports support that $F L G$ variants are unique in each population $[4,5]$. The one variant studied by Chauhan et al. [3], is previously reported in European and Japanese cohorts but not in Chinese or Taiwanese population. Though they report an estimated $48.8 \%$ cases with AA or Aa genotype and $44.4 \%$ Aa in controls, another study by Handa et al. [6] from a similar geographical region of the country report no carrier of R501X variation. Could the patient phenotype contribute to this variance? Younger patients with predominant asthma with or without dermatitis in the current study of Chauhan et al., vs. older patients with hand eczema reported by Handa et al. [6].

Monogenic ichthyosis vulgaris due to mutations in FLG differs from the concept of susceptibility being examined in patients with disorders contributed by the atopic march. Testing for only the common reported variants of other populations for allele frequencies would result in under reporting of genetic contribution in the Indian population that in itself is so diverse. There is a need for larger cohort studies for FLG mutations in defined phenotypes to identify additional risk loci in patients of Indian origin. Though filaggrin Sanger sequencing is the gold standard, new, robust and cheaper sequencing technologies should be used to determine accurately additional genetic contribution to atopy and asthma in India. Larger, genome wide association studies for novel genes and novel variants in known genes can also be deliberated.

$F L G$ testing remains in a research setting till more robust data of variants and their effect on disease causations are understood. The impact on current management and novel 
therapies in pipeline for asthma require greater insight on the implications of $F L G$ variations on disease pathogenesis and drug response [5]. There is a long way ahead but appropriate multicentric, prospective case control studies in various ethnicities in India with appropriate genotype phenotype correlation is the need of the hour.

\section{Compliance with Ethical Standards}

Conflict of Interest None.

\section{References}

1. Paller AS, Spergel JM, Mina-Osorio P, Irvine AD. The atopic march and atopic multimorbidity: many trajectories, many pathways. J Allergy Clin Immunol. 2019;143:46-55.
2. Smith FJD, Irvine AD, Terron-Kwiatkowski A, et al. Loss-offunction mutations in the gene encoding filaggrin cause ichthyosis vulgaris. Nature Genet. 2006;38:337-42.

3. Chauhan A, Panigrahi I, Singh M, Attri SV, Agarwal A, Singh M. Prevalence of filaggrin gene R501X mutation in Indian children with allergic diseases. Indian J Pediatr. 2020. https://doi.org/10.1007/ s12098-020-03231-0.

4. Brunner PM, Guttman-Yassky E. Racial differences in atopic dermatitis. Ann Allergy Asthma Immunol. 2019;122:449-55.

5. Drislane $\mathrm{C}$, Irvine $\mathrm{AD}$. The role of filaggrin in atopic dermatitis and allergic disease. Ann Allergy Asthma Immunol. 2020;124:36-43.

6. Handa S, Khullar G, Pal A, Kamboj P, de D. Filaggrin gene mutations in hand eczema patients in the Indian subcontinent: a prospective case-control study. Contact Dermatitis. 2019;80:359-6.

Publisher's Note Springer Nature remains neutral with regard to jurisdictional claims in published maps and institutional affiliations. 